\title{
An examination of the sustainability of the CXO advantage in U.S. hospitals
}

\author{
Seongwon Choi ${ }^{1}$, Kristine Ria Hearld ${ }^{2}$, Alisha Singh ${ }^{2}$, Geoffrey A. Silvera ${ }^{2}$, William Breen ${ }^{3}$, Nancy Borkowski ${ }^{2}$ \\ ${ }^{1}$ Department of Health Care Administration, Trinity University, San Antonio, TX, USA; ${ }^{2}$ Department of Health Services Administration, University \\ of Alabama at Birmingham, Birmingham, AL, USA; ${ }^{3}$ Honeycomb Management Services, LLC, Memphis, TN, USA \\ Contributions: (I) Conception and design: S Choi, KR Hearld, GA Silvera, N Borkowski; (II) Administrative support: None; (III) Provision of study \\ materials or patients: W Breen; (IV) Collection and assembly of data: A Singh, W Breen; (V) Data analysis and interpretation: S Choi, KR Hearld; (VI) \\ Manuscript writing: All authors; (VII) Final approval of manuscript: All authors. \\ Correspondence to: Seongwon Choi, PhD, MBA. CGC403 1 Trinity Place, San Antonio, TX 78212, USA. Email: schoi2@trinity.edu.
}

Background: The U.S. healthcare industry's shift from fee-for-service to value-based purchasing (VBP) has caused hospitals to strategically adapt. Payers have implemented performance models to incentivize hospitals and other providers to incorporate the concept of patient-centeredness for improving the patient experience. The patient experience domain of the Hospital Consumer Assessment of Healthcare Providers and Systems survey (HCAHPS) is an integral part of the Centers for Medicare \& Medicaid Services' (CMS) hospital VBP program payment adjustment. Many U.S. hospitals have adapted to this new environment by including a new C-suite leader, a Chief Experience Officer (CXO). Recent research has demonstrated an advantage for those hospitals that employ a full-time CXO in terms of HCAHPS performance. The purpose of this study is to determine whether the CXO advantage has a lasting influence on hospital's HCAHPS performance.

Methods: This study expands upon the data used by Breen and colleagues to determine whether the findings of a CXO advantage are consistent over a longer time period (3 years) or if there is a diminishing influence of this new C-suite leader. To answer these questions, a purposive sample of all acute care hospitals operating in three states (California, Florida, and New York, USA) was compiled and combined with HCAHPS performance for 2015 and 2018 ( $\mathrm{n}=608)$.

Results: This study's results confirm the findings of Breen et al. that the CXO advantage with respect to HCAHPS performance are sustained over a significant time period (3 years). Hospitals with a CXO are more likely to have higher HCAHPS scores, and hospitals with CXOs are more likely to have higher scores three years later as well.

Conclusions: Findings of this study provide additional support for the CXO role in U.S. hospitals as CXOs are associated with a sustained advantage in HCAHPS performance. While this study provides further evidence of a $\mathrm{CXO}$ advantage, questions remain regarding how immediately $\mathrm{CXO}$ san positively influence patient experience scores and what organizational policies and practices employed by CXOs are most effective in improving HCAHPS performance.

Keywords: Patient experience; Chief Experience Officer (CXO); Hospital Consumer Assessment of Healthcare Providers and Systems survey (HCAHPS)

Received: 10 September 2021; Accepted: 08 January 2022; Published: 25 September 2022.

doi: $10.21037 /$ jhmhp-21-67

View this article at: https://dx.doi.org/10.21037/jhmhp-21-67 


\section{Introduction}

For over 20 years, the U.S. healthcare system has been tasked with augmenting care delivery to be delivered in a manner that is centered on the needs, preferences, and desires of the patient (1). While efforts to create a more patient centric healthcare system have been ongoing, there has been a paradigm shift within the past decade. The Centers for Medicare \& Medicaid Services (CMS) (2) and other payers have implemented performance models shifting away from fee-for-service reimbursements and towards value-based purchasing (VBP) which incentivize hospitals to provide care in a manner that aligns with the concept of patient-centeredness.

Since the advent of VBP, many hospitals have changed their internal operations to include a position titled, Chief Experience Officer (CXO) within their C-suite to lead patient experience improvement efforts. As Breen et al. (3) describe, the CXO role is to advance the integration of the patient experience as a key factor in both strategic and operational decisions in hospitals. The Cleveland Clinic was the first healthcare organization to introduce the $\mathrm{CXO}$ role in 2003 in an effort to communicate that the patient experience was as equally important as the clinical quality of care they delivered (4). After 2010, recruiting efforts to fill CXO positions increased, and, today, approximately one-third of health systems have a C-suite leader in this position (5).

To date, there has been limited empirical analysis regarding this role and its influence on a hospital performance. Breen and colleagues (3) were one of the first to study (I) the prevalence of the CXO role within the hospital industry, and (II) whether there was an association between an established CXO role and the hospital's patient experience as measured by its Hospital Consumer Assessment of Healthcare Providers and Systems (HCAHPS) scores. In their cross-sectional study (2015), Breen $e t a l$. found that one-third of hospitals studied had a formal CXO position and that those hospitals with a formal CXO role reported significantly higher HCAHPS scores for both overall hospital rating and recommendation of the hospital by patients (3).

The purpose of this study is to determine whether the CXO advantage found by Breen et al. (3) has a lasting influence on hospital's HCAHPS performance. This study expands upon the data used in Breen et al. (3) to determine whether the findings of a CXO advantage are consistent over a longer time period (3 years) or if there is a diminishing influence of this new $\mathrm{C}$-suite leader. Breen and colleagues successfully addressed the association between the CXO position and HCHAPS score using 2015 data. Our study year of 2018 allows us to re-visit Breen et al.'s findings (3) since it provides the time necessary for the continuous maturation of the VBP and CXO position across U.S. hospitals.

The inclusion of this formal role in the hospital's operations requires a considerable investment of organizational resources as well as significant changes to hospital operations. It is thus important to examine the degree to which changes in performance are attributable to this role, and the durability of those changes. To answer these questions, a purposive sample of all acute care hospitals operating in three states (California, Florida, and New York) was compiled and combined with HCAHPS performance for 2015 and $2018(\mathrm{n}=608)$ to determine the sustained influence of CXOs on patient experience performance.

Since 2013, CMS penalizes hospitals when patients report lower satisfaction scores as measured by the HCAHPS survey. Specifically, hospitals are penalized by a proportional reduction in their Medicare reimbursement payments under the hospital VBP program if their scores in the patient experience of care domain are not within a predetermined range as compared to other hospitals and self-improvement scores. In 2013, HCAHPS patient experience scores constituted $30 \%$ of the total incentivebased payments from Medicare's VBP program. Currently, they are weighted at $25 \%$ of the total incentive-based payment. In 2015, 44.5\% of the 3,089 hospitals receiving a hospital VBP program payment adjustment were penalized (6). For fiscal year 2020, CMS reported that it will pay $\$ 1.9$ billion in incentive payments to hospitals under the VPB Program (2). Considering that, pre-COVID, most hospitals operate with profit margins under two percent, these incentives can have a significant impact on hospital's financial sustainability.

Strategic adaptation theory (SAT) guided this research study. SAT describes how changes in the environment drives changes in an organization's internal structure and/or operations. With the industry's push towards VBP in which patient experience influences financial incentives as well as the avoidance of disincentives, hospitals need to strategically adapt their internal structure to fit their new environment. One option for adapting to the new environment is for hospitals to establish a formal CXO role to coordinate and lead improved patient experience efforts. 
The application of SAT is well suited for explaining the impetus for hospitals to establish and to sustain a CXO role within their organizations. As Kimberly and Zajac (7) pointed out, strategic adaption is the relationship between organizations and their environments and seeks to explain an entity's behavior in terms of how it responds to its changing environment. Bruns and Stalker (8) and Lawrence and Lorsch (9) introduced the concept that different environments require different structures, procedures and/or processes not only for an organization's survival but to prosper as well. Zajac and Shortell (10), Zajac and Kraatz (11), and Worley, Cummings and Mobley (12) examined how organizations adapted their strategies to "fit" environmental changes. In other words, organizations need to strategically react to changes within their environments to sustain a good fit.

\section{Methods}

\section{Data}

The sample population in this study were acute care hospitals that operated in three states, California, Florida, and New York, in 2015 and 2018. The sample states were used in the earlier study and were chosen to represent a varied geographic and service sample. After addressing observations with missing values, the final sample resulted in two groups, one that had an established and distinct CXO role $(2015: \mathrm{n}=160 ; 2018: \mathrm{n}=148)$ and another group in which there was no established CXO role or if those duties were associated with another role $(2015: \mathrm{n}=502 ; 2018$ : $\mathrm{n}=460)$. Variables related to patient experience were obtained from the Agency for Healthcare Research and Quality HCAHPS for 2018. Hospital characteristics data was extracted from the American Hospital Association (AHA) survey database for the year 2018. The market factors for each facility were extracted from the 2018 Area Health Resource File (AHRF). The study was conducted in accordance with the Declaration of Helsinki (as revised in 2013). The authors assert that all procedures contributing to this work comply with the ethical standards of the ICMJE national guidelines on human experimentation and has been approved by the appropriate committees at our institution. Given the nature of this study, the institution review board/ethics committee did not require HIPAA Authorization, Assent, and Parental Permission under Exempted criterion as Not Human Subjects Research designation.

\section{Patient experience}

Patient experience was assessed using two HCAHPS items that gather patient's assessment on (I) overall hospital rating and (II) willingness to recommend the hospital. For the overall hospital rating HCAHPS item, we extracted the variable reporting the percentage of respondents that answered 9 or 10 to the question that asked, "Using any number from 0 to 10 , where 0 is the worst hospital possible and 10 is the best hospital possible, what number would you use to rate this hospital during your stay?". For the variable estimating the willingness to recommend the hospital, we obtained the variable reporting the summarized percentage of respondents that answered, "probably yes" and "definitely yes" to the HCAHPS item asking the patient if they would recommend the hospital to their friends and family.

\section{CXO}

We created a binary indicator reflecting whether the hospital had an established formal full-time CXO role at the facility $(=1)$ or not $(=0)$ based on information gathered by visiting the web pages of 608 hospitals. The data was collected in two steps. First, based on the 2021 Breen et al.'s study, a researcher visited the web sites of hospitals that had previously established the formal CXO role and identified if the hospital still maintained the CXO position. Next, a second stage of web searches was performed to identify whether the hospitals that did not have a formal CXO role in 2015 had established the CXO position thereafter.

\section{Organizational characteristics}

A number of studies have investigated the organizational characteristics of hospitals that are high performing on the various domains of the HCAHPS survey $(13,14)$. Understanding associations between hospital characteristics and HCAHPS scores, we controlled for organization level variables reflecting the hospital bed size, ownership type, teaching status, and nurse staffing ratio. We created a variable to reflect the hospital's bed size that is consisted of four categories: less than 100 beds (referent), 100-299 beds, 300-499 beds, and greater than 499 beds. The hospital's teaching status was reflected by a dichotomous variable that indicated whether the hospital was a teaching hospital or not. Hospital's ownership type was measured as a categorical variable consisted of three categories: not-for-profit, for-profit, and governmental (referent). Lastly, nurse staffing ratio was measured as the 
number of nurse hours per adjusted patient day.

\section{Market characteristics}

Previously, Lehrman et al. (15) examined market factors associated with higher HCAHPS scores and found that hospitals in more urban locations with higher per capita income tended to score better on HCAHPS results. The market factors included in this study are the following: population density, percent minority in market population, per capita income, and market competition. Population density was measured as a dichotomous variable: urban vs. not urban (referent). Percent minority in the market population was measured as the percentage of county residents who are non-White. Per capita income was measured as a continuous variable that captured the average per capita income of the county. Finally, the level of competition was determined as the Herfindahl-Hirschman Index (HHI), with lower numbers reflecting higher market competition and higher numbers reflecting lower market competition. A HHI was calculated based on the Health Service Areas for hospitals.

\section{Statistical analysis}

The data were analyzed using Stata Version 16 and employed logistic regression and ordinary least squares (OLS) regressions (2015: $\mathrm{n}=662 ; 2018: \mathrm{n}=608)$. We conducted two regression analyses. First, we analyzed the relationship between hospitals with a CXO position and organizational and market characteristics using a logistic regression analysis. Second, we employed OLS regression to analyze the relationship between the hospital's patient experience ratings and the $\mathrm{CXO}$ position, controlling for organizational characteristics and market factors.

\section{Results}

Univariate and bivariate results are displayed in Table 1. Descriptive statistics compared non-CXO hospitals to CXO hospitals, and tests of significance were conducted as appropriate (chi-square for categorical variables and $t$ tests for continuous variables). Approximately one-quarter of the hospitals had a formal CXO position (2015: 24.2\%; 2018: $24.3 \%)$. The percent of patients reporting high overall hospital rating scores (or 9 or 10) was significantly higher for hospitals with a CXO position compared to hospitals without a formal CXO position (2015: 68.5\% vs. $66.3 \%$;
2018: $69.6 \%$ vs. $67.8 \%$, respectively, $\mathrm{P}<0.05)$. Similarly, hospitals with a CXO position had a higher percentage of patients reporting answering "probably yes or definitely yes" to whether they would recommend the hospital, compared to hospitals without a CXO position (2015: 93.8\% vs. $92.1 \%$; 2018: $93.7 \%$ vs. $92.9 \%$, respectively, $\mathrm{P}<0.01)$. Regardless of having CXO position and year, approximately $50 \%$ of the hospitals were the size of 100-299 beds. In 2015, 56.3\% of the CXO hospitals were teaching hospitals and this increased to $60.1 \%$ in 2018 . Two-thirds of the hospitals with a CXO position were notfor-profits hospitals compared to $60 \%$ of the hospitals without a CXO (2015: $65.0 \%$ vs. $58.8 \%$; 2018: $66.2 \%$ vs. $59.8 \%$ ) and approximately $30 \%$ of the hospitals with a CXO were for-profit hospitals compared to $21 \%$ of the hospitals without a CXO (2015: 29.4\% vs. 20.3; 2018: 29.7\% vs. 20.9, $\mathrm{P}<0.001$ ). Approximately $87 \%$ of the hospitals without a $\mathrm{CXO}$ were located in urban areas whereas over $93 \%$ of the hospitals with a CXO were located in urban areas (2015: 93.1\% vs. $86.9 \%$; 2018: $93.2 \%$ vs. $87.4 \%$, respectively, $\mathrm{P}<0.05)$. Hospitals with a CXO position had significantly higher average per capita income in the surrounding areas compared to hospitals without a CXO position (2015: $\$ 52,520$ vs. $\$ 47,529 ; 2018$ : $\$ 63,596$ vs. $\$ 58,480$, respectively, $\mathrm{P}<0.05)$. The mean HHI in 2015 was 0.60 regardless of having a CXO position, and in 2018 was significantly lower (i.e., higher market competition) for hospitals with a CXO position compared to hospitals without a CXO officer (0.57 vs. 0.65).

We employed logistic regressions to evaluate the market and organization characteristics statistically associated with the CXO position (Table 2). Not-for-profit hospitals were associated with over 4.1 times and 6.1 times higher odds of having a CXO officer compared to governmental hospitals (2015: $\mathrm{OR}=4.11 ; 2018: \mathrm{OR}=6.0 \% ; \mathrm{P}<0.001)$ in 2015 and 2018 respectively. For-profit hospitals were associated with almost 7 times higher odds of having a CXO officer and 8 times higher odds of having a CXO officer compared to governmental hospitals (2015: OR $=6.82 ; 2018$ : $\mathrm{OR}=8.5 \%$; $\mathrm{P}<0.001)$. In 2015, hospitals located in areas with higher per capita income were associated with higher odds of having a CXO officer $(\mathrm{OR}=1.01, \mathrm{P}<0.05)$; whereas, in 2018, hospitals located in areas with higher market competition were associated with lower odds of having a formal CXO officer $(\mathrm{OR}=0.42, \mathrm{P}<0.01)$.

Table 3 displays the results of ordinary least squares regression examining the relationship between the CXO 
Table 1 Bivariate associations between sample characteristics and $\mathrm{CXO}$ position

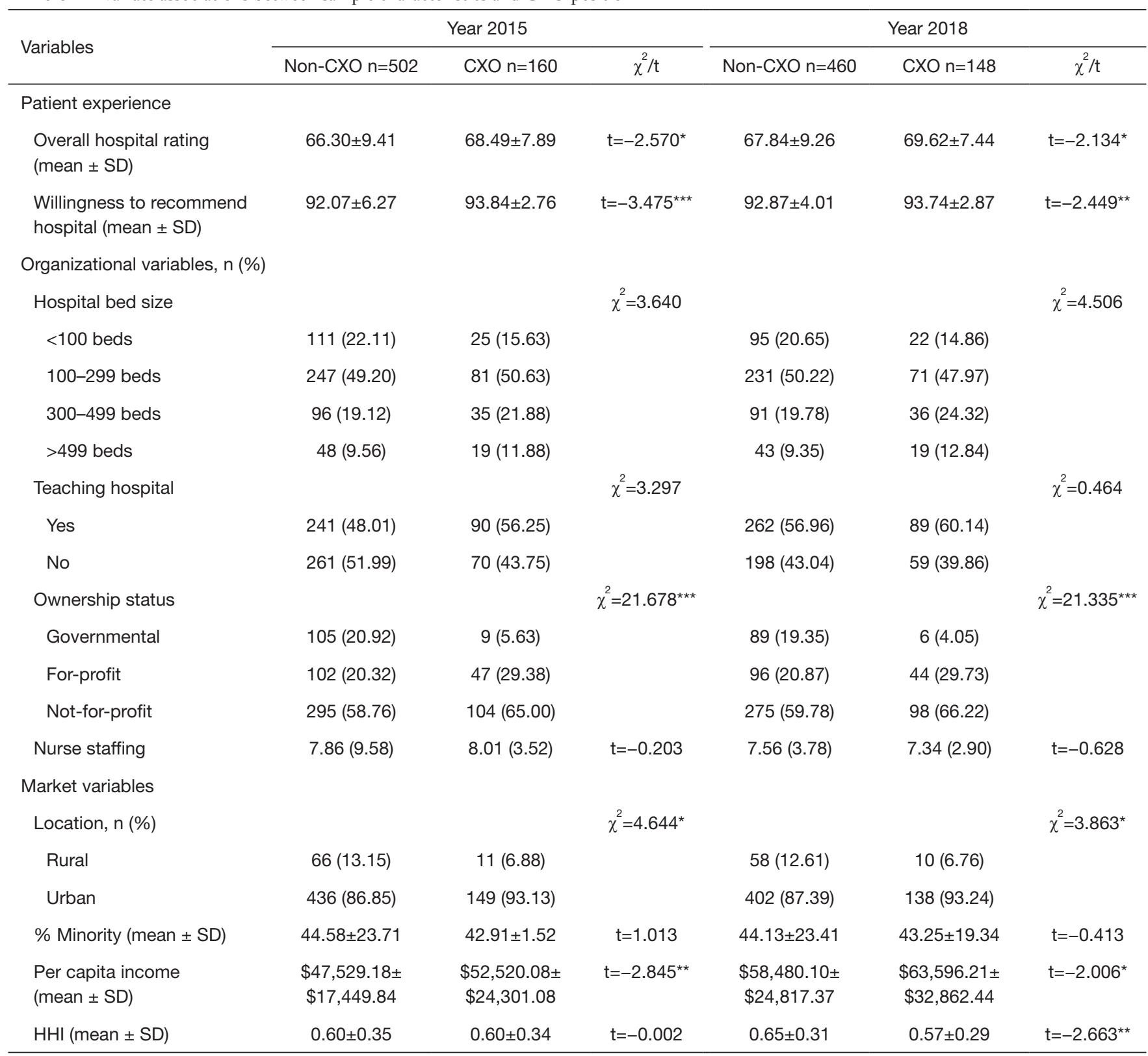

${ }^{\star \star \star} \mathrm{P}<0.001,{ }^{* \star} \mathrm{P}<0.01,{ }^{\star} \mathrm{P}<0.05 . \mathrm{N}=662$ for $2015, \mathrm{~N}=608$ for 2018. CXO, Chief Experience Officer; HHI, Herfindahl-Hirschman Index; SD, standard deviation.

position and two patient experience HCAHPS measures in 2015 and 2018: overall hospital rating and willingness to recommend the hospital.

\section{Overall bospital rating}

Compared to hospitals without a CXO officer, hospitals with a CXO officer were associated with significantly higher percentages of patients reporting high overall hospital rating scores (or 9 or 10) compared to hospitals without a CXO officer (2015: $\mathrm{b}=2.06, \mathrm{P}<0.05 ; 2018: \mathrm{b}=2.65, \mathrm{P}<0.01)$. This confirms the sustainability of the CXO advantage in regards to overall hospital rating.

There were also a number of interesting significant associations found between the control variables and HCAHPS ratings. Relative to hospitals with under 100 beds, 
Table 2 Logistic regression examining correlates of CXO position

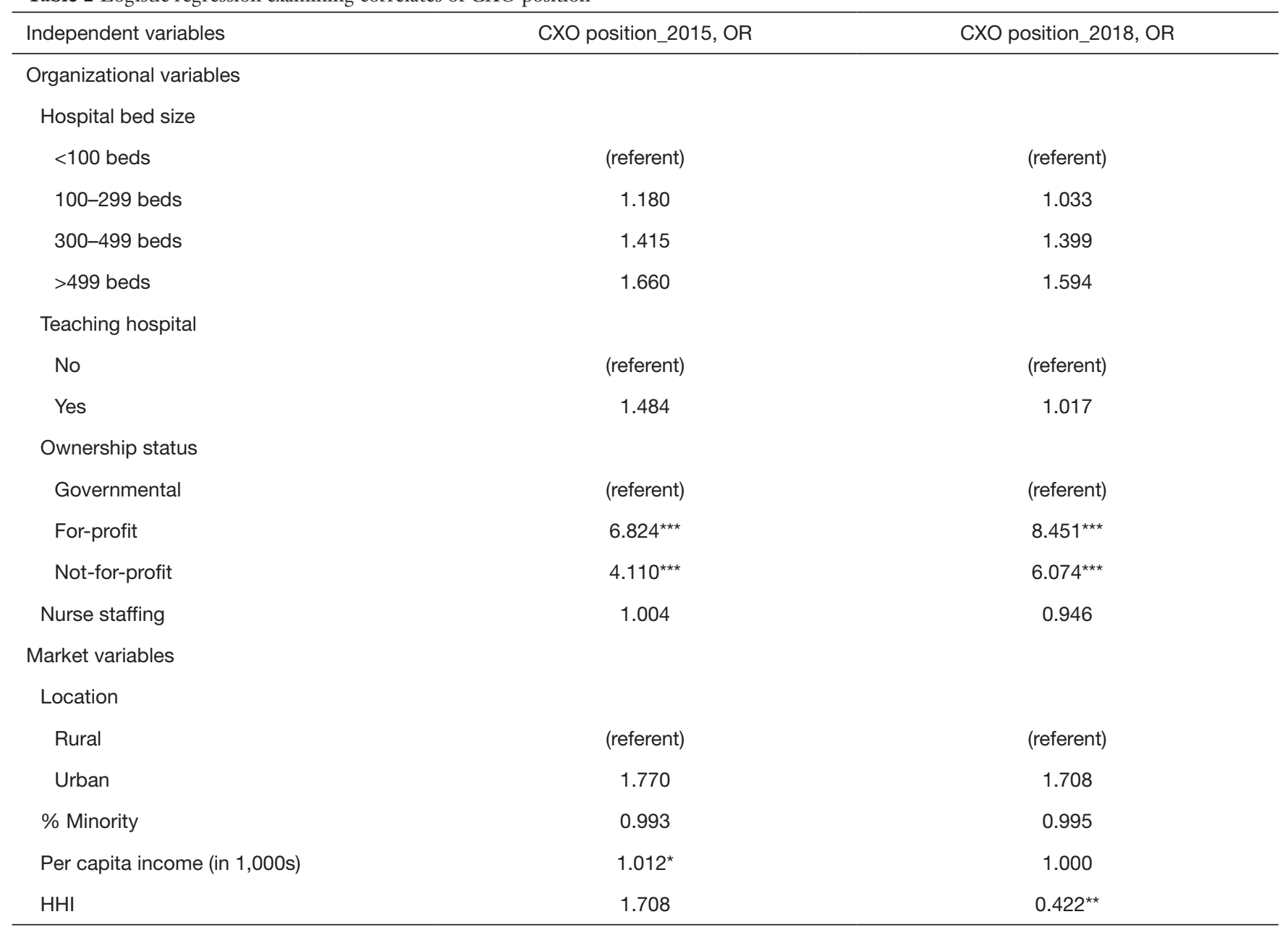

${ }^{\star * *} \mathrm{P}<0.001,{ }^{* \star} \mathrm{P}<0.01,{ }^{*} \mathrm{P}<0.05 . \mathrm{N}=662$ for $2015, \mathrm{~N}=608$ for 2018. CXO, Chief Experience Officer; OR, odds ratio; HHI, HerfindahlHirschman Index.

hospitals with greater numbers of beds were associated with lower overall hospital rating scores $(\mathrm{P}<0.001)$. Specifically, in 2015, hospitals with 100-299 beds were associated with $4.2 \%$ fewer patients reporting high overall hospital rating scores $(\mathrm{P}<0.001)$, hospitals with $300-499$ beds were associated with $3.5 \%$ fewer patients reporting high overall hospital rating scores $(\mathrm{P}<0.01)$, and hospitals with more than 499 beds were associated with $4.7 \%$ fewer patients reporting high overall hospital rating scores compared to hospitals with less than 100 beds $(\mathrm{P}<0.01)$. Likewise, in 2018, hospitals with 100-299 beds were associated with $5.0 \%$ fewer patients reporting high overall hospital rating scores $(\mathrm{P}<0.001)$, hospitals with $300-499$ beds were associated with $4.4 \%$ fewer patients reporting high overall hospital rating scores $(\mathrm{P}<0.001)$, and hospitals with more than 499 beds were associated with $5.3 \%$ fewer patients reporting high overall hospital rating scores compared to hospitals with less than 100 beds $(\mathrm{P}<0.001)$. In 2015 forprofit hospitals were associated with $2.6 \%$ fewer patients reporting high overall hospital rating scores compared to governmental hospitals $(\mathrm{P}<0.05)$. Interestingly, in 2015 notfor-profit hospitals were associated with higher percentages of patients reporting high overall hospital rating scores compared to governmental hospitals $(\mathrm{b}=3.21, \mathrm{P}<0.01)$ whereas in 2018, for-profit hospitals were associated with $5.1 \%$ fewer patients reporting high overall hospital rating scores compared to governmental hospitals $(\mathrm{P}<0.001)$. Increases in nurse staffing was associated with higher percentages of patients reporting high overall hospital rating scores (2015: $\mathrm{b}=0.21, \mathrm{P}<0.001 ; \mathrm{b}=0.70, \mathrm{P}<0.001)$. 
Table 3 Relationship of HCAHPS on patient experience HCAHPS ratings, OLS regression

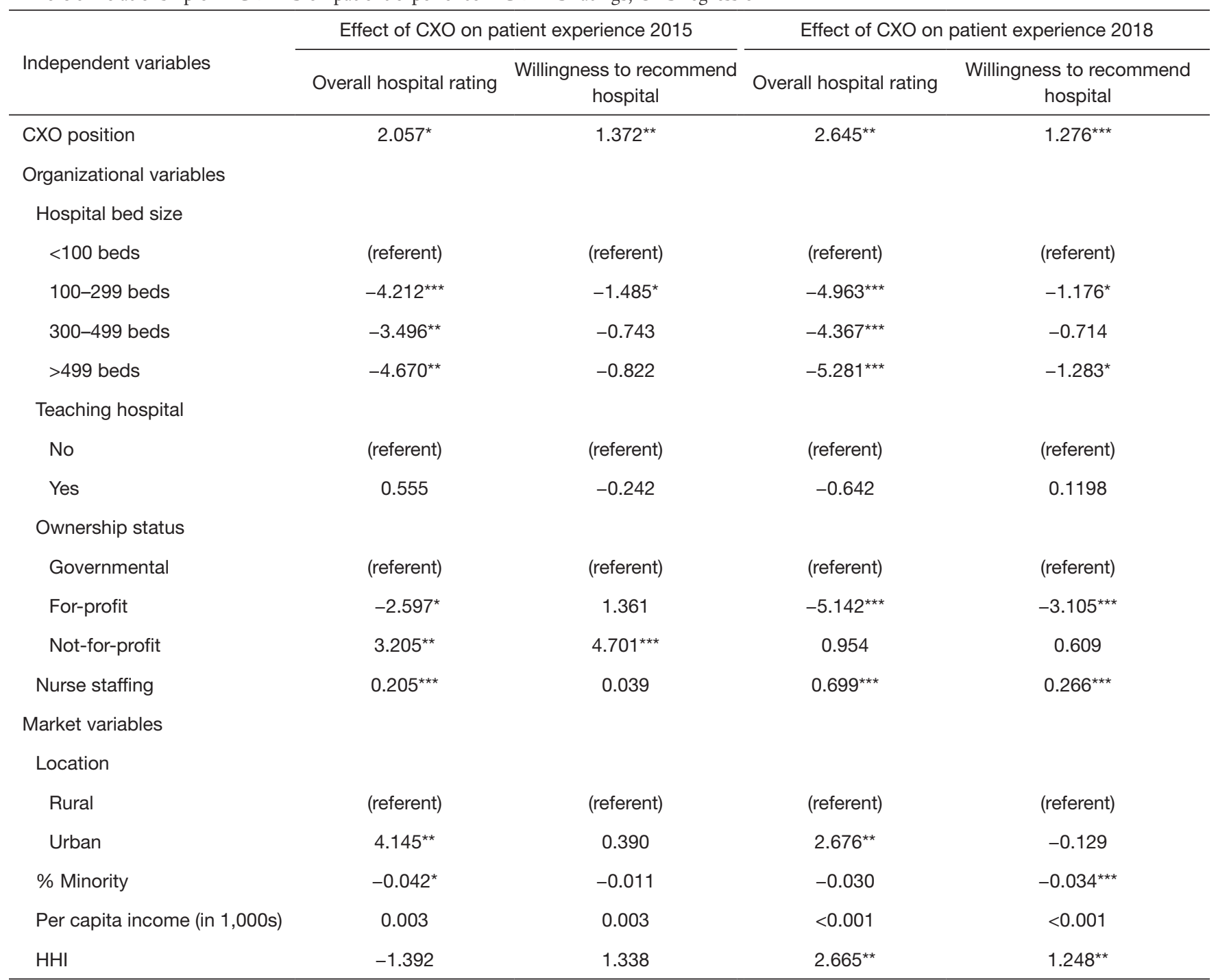

${ }^{* * *} \mathrm{P}<0.001,{ }^{* \star} \mathrm{P}<0.01,{ }^{*} \mathrm{P}<0.05 . \mathrm{N}=662$ for $2015, \mathrm{~N}=608$ for 2018. HCAHPS, Hospital Consumer Assessment of Healthcare Providers and Systems survey; OLS, ordinal least square; CXO, Chief Experience Officer; HHI, Herfindahl-Hirschman Index.

In 2015, urban hospitals were associated with $4.15 \%$ more patients reporting high overall hospital rating compared to rural hospitals, and in 2018, 2.7\% more patients reporting high overall hospital rating scores compared to rural hospitals $(\mathrm{P}<0.01)$. In 2015, hospitals located in areas with higher percentages of minority population were associated with lower overall hospital ratings $(b=-0.04, \mathrm{P}<0.05)$ and in 2018 , hospitals located in areas with higher market concentration were associated with $2.7 \%$ more patients reporting high overall hospital rating scores $(\mathrm{P}<0.01)$.

\section{Willingness to recommend hospital}

Relative to hospitals without a CXO officer, hospitals with a CXO officer were also associated with significantly higher percentages of patients reported answering "probably yes or definitely yes" to whether they would recommend the hospital (2015: $\mathrm{b}=1.37, \mathrm{P}<0.01 ; 2018: \mathrm{b}=1.28, \mathrm{P}<0.001$ ), further confirming the sustainability of the CXO advantage.

Among the control variables, several of the hospital characteristics were significantly associated with the HCAHPS question regarding willingness to recommend 
the hospital. Relative to hospitals with less than 100 beds, hospitals in 2015 with 100-299 beds were associated with $1.49 \%$ fewer patients reporting "probably yes or definitely yes" to whether they would recommend the hospital $(\mathrm{P}<0.05)$ and $1.18 \%$ fewer patients in 2018 , relative to hospitals with less than 100 beds $(\mathrm{P}<0.05)$. Likewise, compared to hospitals with less than 100 beds, hospitals with over 499 beds were associated with $1.3 \%$ fewer patients reporting "probably yes or definitely yes" to whether they would recommend the hospital $(\mathrm{P}<0.05)$, but this association is found only for 2018. Relative to governmental hospitals, not-for-profit hospitals in 2015 were associated with $4.7 \%$ more patients reporting "probably yes or definitely yes" to whether they would recommend the hospital $(\mathrm{P}<0.001)$, whereas in 2018 this association was not found. Instead, in 2018, for-profit hospitals were associated with $3.11 \%$ fewer patients reporting "probably yes or definitely yes" to whether they would recommend the hospital $(\mathrm{P}<0.001)$. In 2018 , increases in nurse staffing was associated with higher percentages of patients reporting "probably yes or definitely yes" to whether they would recommend the hospital $(b=0.3, \mathrm{P}<0.001)$ and hospitals located in areas with higher percentages of minority population were associated with lower percentage of patients reporting "probably yes or definitely yes" to whether they would recommend the hospital $(\mathrm{b}=-0.03, \mathrm{P}<0.001)$. Higher market concentration was associated with significantly higher percentages of patients reporting probably yes or definitely yes" to whether they would recommend the hospital $(b=1.25, \mathrm{P}<0.05)$.

\section{Discussion}

As previously noted, we wanted to explore if our 2018 findings would be similar with the 2015 findings of Breen and colleagues (3), more specifically if hospitals that continue to employ a formal full-time CXO role report higher HCAHPS scores than hospitals without this C-suite position. Our findings are consistent with Breen et al.'s (3) study 3 -year afterwards. Both studies find that hospitals that maintain a formal CXO role have significantly higher rating for overall experience and willingness to recommend HCAHPS scores. Based on the findings of this study, it can be inferred that hospitals receive a return on their investments in establishing a formal CXO role and that this investment has residual influence on patient experiences.

In 2015, HHI was not significantly associated with hospitals having a CXO position whereas in 2018, hospitals in areas with higher market competition were associated with the CXO position. On the other side of the coin, in 2015 , increases in per capita income were associated with increased odds of hospitals having a CXO position and in 2018 per capita income is not significantly associated hospitals having a CXO position (3). We did not find differences regarding organizational characteristics between the two studies and only two differences, HHI and per capita income, were noted regarding market factors for hospitals having a CXO role.

Regarding HCAHPS scores, we found a few differences between the two studies among both organizational characteristics and market factors but only one with significance. In 2018, hospitals with over 499 beds were associated with $1.3 \%$ fewer patients reporting "probably yes or definitely yes" to whether they would recommend the hospital $(\mathrm{P}<0.05)$. In 2015 , there was no significant difference between hospitals with less than 100 beds and those with over 499 beds in willingness to recommend the hospital. In contrast with 2015, in 2018, there was no significant associations between hospital's not-for-profit status and higher HCHAPS scores.

\section{Practice application}

Delivering a high-quality patient experience is both a clinical and business requirement in today's healthcare environment. As noted by Manary, Staelin, Kosel, Schulman and Glickman (16) the patient experience is fundamental to care delivery for improving quality and lowering costs. Studies have shown a relationship between positive patient experiences and greater patient safety, employee satisfaction, and physician engagement, and lower utilization of resources and readmission rates (17). In addition, the patient experience can be viewed as a differentiator within a highly competitive market (18). As such, there are varying outcomes, beyond reimbursement implications, to justify continued investment in a patient experience leader position.

\section{Limitations}

As with all studies, there are noted limitations. We were not able to provide information regarding the length of tenure or characteristics of individuals (i.e., clinical versus non-clinical) holding the $\mathrm{CXO}$ position within our study population. This information could provide a broader understanding into patient experience results. Future studies may want to include the financial position of the hospitals in 
relation to their patient experience scores. Finally, although the three states chosen for this study are among the most populous in the nation and have differing levels of managed care penetration, which influences consumer engagement, future researchers may wish to examine hospitals in different states or nationally for better generalizability.

\section{Conclusions}

This study adds to the literature to increase our knowledge regarding the organizational benefits of a relatively new C-suite position within the healthcare industry, the CXO. This study provides additional insight into the organizational characteristics and the external environment of the hospitals that have formalized the CXO role as an adaptive strategy to the changing industry. Based on strategic adaptation theory, this study offers a perspective to support the organizational investments required to improve the patient experience. The findings of this study are timely. Although there was a slowdown period with some hospitals either eliminating the position or combining the responsibilities with another executive role (5), currently the CXO position is one of the fastest growing C-suite positions (19). These noted changes in the adoption of the $\mathrm{CXO}$ position may reflect an underlying ambiguity about the role's effectiveness. This study provides further evidence of the CXO's effectiveness in improving and sustaining better patient experience performance as it confirms a CXO advantage germane to HCAHPS performance and it shows that the $\mathrm{CXO}$ advantage is enduring. Future research should seek to determine the underlying mechanisms (i.e., policies and practices) employed by CXOs which enable them to be successful with patient experience improvement efforts.

\section{Acknowledgments}

Funding: None.

\section{Footnote}

Provenance and Peer Review: This article was commissioned by the editorial office, Fournal of Hospital Management and Health Policy for the series "Shaping Tomorrow's Healthcare Systems: Key Stakeholders' Expectations and Experiences”. The article has undergone external peer review.

Data Sharing Statement: Available at https://jhmhp. amegroups.com/article/view/10.21037/jhmhp-21-67/dss

Conflicts of Interest: All authors have completed the ICMJE uniform disclosure form (available at https://jhmhp. amegroups.com/article/view/10.21037/jhmhp-21-67/coif). The series "Shaping Tomorrow's Healthcare Systems: Key Stakeholders' Expectations and Experiences" was commissioned by the editorial office without any funding or sponsorship. NB served as the unpaid Guest Editor of the series. The authors have no other conflicts of interest to declare.

Ethical Statement: The authors are accountable for all aspects of the work in ensuring that questions related to the accuracy or integrity of any part of the work are appropriately investigated and resolved. The study was conducted in accordance with the Declaration of Helsinki (as revised in 2013). The authors assert that all procedures contributing to this work comply with the ethical standards of the ICMJE national guidelines on human experimentation and has been approved by the appropriate committees at our institution. Given the nature of this study, the institution review board/ethics committee did not require HIPAA Authorization, Assent, and Parental Permission under Exempted criterion as Not Human Subjects Research designation.

Open Access Statement: This is an Open Access article distributed in accordance with the Creative Commons Attribution-NonCommercial-NoDerivs 4.0 International License (CC BY-NC-ND 4.0), which permits the noncommercial replication and distribution of the article with the strict proviso that no changes or edits are made and the original work is properly cited (including links to both the formal publication through the relevant DOI and the license). See: https://creativecommons.org/licenses/by-nc-nd/4.0/.

\section{References}

1. Institute of Medicine (US) Committee on Quality of Health Care in America. Crossing the Quality Chasm: A New Health System for the 21st Century. Washington, DC: National Academies Press (US), 2001.

2. Centers for Medicare \& Medicaid Services. CMS Hospital Value-Based Purchasing Program Results for Fiscal Year 2020. Available online: https://www.cms.gov/newsroom/ fact-sheets/cms-hospital-value-based-purchasingprogram-results-fiscal-year-2020 
3. Breen W, Choi S, Herald KR, et al. The association between an established Chief Experience Officer role and hospital patient experience scores. Patient Exp J 2021;8:69-76.

4. Carlson B. The rise of the chief experience officer. Physician Leadersh J 2015;2:16-21.

5. Castellucci M. Shared experience: Use of CXO role fades as other leaders take on responsibilities. Modern Healthcare 2019;49:18.

6. Kahn CN III, Ault T, Potetz L, et al. Assessing Medicare's hospital pay-for-performance programs and whether they are achieving their goals. Health Affairs. 2015;34:1281-8.

7. Kimberly JR, Zajac EJ. Strategic adaptation in health care organizations: implications for theory and research. Med Care Rev 1985;42:267-302.

8. Bruns T, Stalker G. The management of innovation. London: Tavistock Publications, 1961:120-2.

9. Lawrence PR, Lorsch JW. Differentiation and integration in complex organizations. Adm Sci Q 1967;12:1-47.

10. Zajac EJ, Shortell SM. Changing generic strategies: Likelihood, direction, and performance implications. Strateg Manag J 1989;10:413-30.

11. Zajac EJ, Kraatz MS. A diametric forces model of strategic change: Assessing the antecedents and consequences of restructuring in the higher education industry. Strateg Manag J 1993;14:83-102.

doi: 10.21037/jhmhp-21-67

Cite this article as: Choi S, Hearld KR, Singh A, Silvera GA, Breen W, Borkowski N. An examination of the sustainability of the CXO advantage in U.S. hospitals. J Hosp Manag Health Policy 2022;6:26.
12. Worley CG, Cummings TG, Mobley F. The dynamics of strategic change in hospitals: managed care strategies, organization design, and performance. Public Adm Manag 2000;5:1-27.

13. Chatterjee P, Joynt KE, Orav EJ, et al. Patient experience in safety-net hospitals: implications for improving care and value-based purchasing. Arch Intern Med 2012;172:1204-10.

14. Richter JP, Muhlestein DB. Patient experience and hospital profitability: Is there a link? Health Care Manage Rev 2017;42:247-57.

15. Lehrman WG, Elliott MN, Goldstein E, et al. Characteristics of hospitals demonstrating superior performance in patient experience and clinical process measures of care. Med Care Res Rev 2010;67:38-55.

16. Manary M, Staelin R, Kosel K, et al. Organizational characteristics and patient experiences with hospital care: a survey study of hospital chief patient experience officers. Am J Med Qual 2015;30:432-40.

17. Meyer MA. Qualifications and Skills Required for Patient Experience Positions. J Patient Exp 2020;7:1535-42.

18. Barden A, Giammarinaro N, Bashkin N, et al. Development and reliability of a patient experience inventory tool for hospitals. Patient Exp J 2020;7:100-4.

19. Caruso M. Diversity a 'vital' part of the C-suite. Mod Healthc 2021;50:45. 